\title{
60. On Closed Mappings
}

\author{
By Sitiro HANAI \\ Osaka University of the Liberal Arts and Education \\ (Comm. by K. KunUGI, M.J.A., April 12, 1954)
}

If $S$ and $E$ are $T_{1}$-spaces, a single-valued mapping $f(S)=E$ is said to be closed provided that the image of every closed set in $S$ is closed in $E$. It is interesting to know how the topology of $E$ is affected by the topology of $S$ under $f$. Concerning this question, G. T. Whyburn and A. V. Martin have recently investigated and obtained some results. ${ }^{12}$

In this note, we will consider the case when the topology of $E$ affected by the topology (under some restrictions) of $S$ under $f$ becomes metrizable.

1. We will firstly prove the following

Theorem 1. Let $S$ be a perfectly separable Hausdorff space and let $E$ a compact space. ${ }^{2}$ If $f(S)=E$ is a closed mapping such that $f^{-1}(p)$ is compact for every point $p$ of $E$, then $E$ is a separable metric space.

To establish this theorem, we prove the following lemmas.

Lemma 1. Let $S$ be a perfectly separable Hausdorff space. If $f(S)=E$ is a closed continuous mapping such that $f^{-1}(p)$ is compact for every point $p$ of $E$, then $E$ is perfectly separable.

Proof. Let $\left\{U_{n}\right\}(n=1,2,3, \ldots)$ be a countable basis of open sets of $S$. For each finite subset $\left(n_{1}, n_{2}, \ldots, n_{m}\right)$ of $(1,2,3, \ldots)$, let $\left(\sum_{i=1}^{m} U_{n_{i}}\right)_{0}$ be the union of all $f^{-1}(p)$ such that $\sum_{i=1}^{m} U_{n_{i}} \supset f^{-1}(p)$. Then $\left(\sum_{i=1}^{m} U_{n_{i}}\right)_{0}$ is an open inverse set, and the family $\left\{\left(\sum_{i=1}^{m} U_{n_{i}}\right)_{0}\right\}$ of all such sets is evidently countable.

Now let $O$ be an open set of $E$ and $p \in O$, then $f^{-1}(O) \supset f^{-1}(p)$ and $f^{-1}(O)$ is open in $S$ because $f$ is continuous. Then $f^{-1}(O)=\sum_{j=1}^{\infty} U_{n_{j}}$ where $\left\{U_{n_{j}}\right\} \subset\left\{U_{n}\right\}(n=1,2,3, \ldots)$. Since $f^{-1}(p)$ is compact, there exists a finite subset $\left\{U_{n_{k}}\right\}(k=1,2, \ldots, l)$ of $\left\{U_{n_{j}}\right\}(j=1,2,3, \ldots)$ such that $\sum_{k=1}^{l} U_{n_{b}} \supset f^{-1}(p)$, hence $\left(\sum_{k=1}^{l} U_{n_{k}}\right)_{0} \supset f^{-1}(p)$. As $f$ is closed and

1) G. T. Whyburn: Open and closed mappings, Duke Math. Jour., 17, 69-74 (1950). A. V. Martin: Decompositions and quasi-compact mappings, (abstract), Bull. Amer. Math. Soc., 59, 397 (1953).

2) We use "compact" in the sense of "bicompact". 
continuous, $f\left\{\left(\sum_{k=1}^{l} U_{n_{k}}\right)_{0}\right\}$ is open in $E$. Hence the family of open sets $\left[f\left\{\left(\sum_{i=1}^{m} U_{n_{i}}\right)_{0}\right\}\right]$ is a countable basis of open sets of $E$. Thus we get the lemma.

Lemma 2. Let $S$ be a Hausdorff space and let $E$ a compact space. If $f(S)=E$ is a closed mapping such that $f^{-1}(p)$ is compact for each point $p$ of $E$, then $f$ is continuous.

Proof. Suppose on the contrary that $f$ is not continuous. Then there exists a point $a \in S$ at which $f$ is not continuous. Let $\mathfrak{F}$ be the filter of neighborhoods of $a$ and $\widetilde{F}^{\prime}$ be the filter whose base is $f(\Re)$. Then $\mathfrak{F}^{\prime}$ does not converge to $f(a) \equiv p$. Hence there exists an open neighborhood $V(p)$ such that $V(p) \bar{\epsilon} \mathfrak{F}^{\prime}$. Let $\Phi^{\prime}$ be an ultrafilter which contains $\widetilde{F}^{\prime}$, then $C V(p) \bar{\epsilon} \Phi^{\prime}$ where $C V(p)$ denotes the complement of $V(p)$. Since $E$ is compact by assumption, $\Phi^{\prime}$ converges to a point $q$. Then $p \neq q$ because $C V(p) \in \Phi^{\prime}$, and hence $a \bar{\epsilon} f^{-1}(q)$. As $f^{-1}(q)$ is compact and $S$ is a Hausdorff space, there exist open neighborhoods $U(a)$ and $U\left\{f^{-1}(q)\right\}$ of $a$ and $f^{-1}(q)$ respectively such that $U(a) U\left\{f^{-1}(q)\right\}=\phi$. Then $\overline{U(a)} f^{-1}(q)=\phi$ and hence $q \bar{\epsilon} f\{\overline{U(\alpha)}\}$.

On the other hand, since $f\{U(\alpha)\} \in \Phi^{\prime}$ and $\Phi^{\prime} \rightarrow q$, we have $V(q)$ $f\{U(a)\} \neq \phi$ for every open neighborhood $V(q)$ of $q$. Hence $q \in \overline{f\{U(a)\}}$. Because $f$ is closed, we have $\overline{f\{U(a)\}} \subset f\{\overline{U(a)}\}$, which contradicts the fact that $q \bar{\epsilon} f\{\overline{U(a)}\}$.

Proof of Theorem 1. By lemmas 1 and 2, it is evident that $E$ is perfectly separable. To prove that $E$ is metrizable, we have only to prove that $E$ is a Hausdorff space because $E$ is a compact space. Let $p$ and $q$ be any distinct two points of $E$. Then $f^{-1}(p) f^{-1}(q)=\phi$, and $f^{-1}(p)$ and $f^{-1}(q)$ are compact sets by assumption. Since $S$ is a Hausdorff space, there exist open neighborhoods $U\left\{f^{-1}(p)\right\}$ and $U\left\{f^{-1}(q)\right\}$ of $f^{-1}(p)$ and $f^{-1}(q)$ respectively such that $U\left\{f^{-1}(p)\right\}$ $U\left\{f^{-1}(q)\right\}=\varnothing$.

As $f$ is closed continuous, there exist open inverse sets $U_{0}\left\{f^{-1}(p)\right\}$ and $U_{0}\left\{f^{-1}(q)\right\}$ such that $U\left\{f^{-1}(p)\right\} \supset U_{0}\left\{f^{-1}(p)\right\} \supset f^{-1}(p)$ and $U\left\{f^{-1}(q)\right\} \supset U_{0}\left\{f^{-1}(q)\right\} \supset f^{-1}(q)$. Hence $E$ is a Hausdorff space.

Remark. Theorem 1 is also proved by use of Lemma 2, Theorem 3 in the following section, and T. Iwamura's theorem. ${ }^{\text {s) }}$

2. In this section, we will consider the case when $S$ is a metric space.

We begin with proving the following:

3) T. Iwamura: Remarks on closed mappings and compactness, Natural Science Report of the Ochanomizu Univ., 1, 6-8 (1951). 
Theorem 2. If $S$ is a metric space and $f(S)=E$ is a closed continuous mapping, then $E$ is a metric space.

Proof. (1) $E$ satisfies the first countability axiom. In fact, let $O^{(n)}(x)(n=1,2,3, \ldots)$ be open spheres with the center $x$ and the radius $\frac{1}{n}$ for each point $x$ of $S$. For any point $p$ of $E$, we have $\sum_{x \in f^{-1}(p)} O^{(x)}(x) \supset f^{-1}(p)$. Since $f$ is closed continuous, in the same way as Lemma 1, we can see that $\left(\sum_{x \in f^{-1}(p)} O^{(n)}(x)\right)_{0}$ is an open inverse set. Let $O_{0}^{(n)}(p) \equiv\left(\sum_{x \in \mathcal{f}^{-1}(p)} O^{(n)}(x)\right)_{0}$ and let $G^{(n)}(p) \equiv f\left\{O_{0}^{(n)}(p)\right\}$, then $G^{(n)}(p)$ is an open neighborhood of $p$.

Now we will prove that $\left\{G^{(n)}(p)\right\}(1,2,3, \ldots)$ is a basis of the neighborhood system of $p$. Let $O(p)$ be any open neighborhood of $p$, then $f^{-1}\{O(p)\}$ is open in $S$ because $f$ is continuous. Let $d$ be the distance between $C f^{-1}\{O(p)\}$ and $f^{-1}(p)$, then $\sum_{x \in f^{-1}(p)} O^{(n)}(x) C f^{-1}\{O(p)\}=\phi$ for an $n$ such that $\frac{1}{n}<d$. Hence $O_{0}^{(n)}(p) \subset f^{-1}\{O(p)\}$. Then we have $G^{(m)}(p) \subset O(p)$. Thus $E$ satisfies the first countability axiom. Further it is easy to see that $G^{(1)}(p) \supset G^{(2)}(p) \supset \cdots$ and $\prod_{n} G^{(n)}(p)=p$.

(2) For any point $p$ of $E$ and any index $n$, there exists some index $m=m(p, n)$ such that $G^{(m)}(p) G^{(m)}(q) \neq \phi$ implies $G^{(m)}(q) \subset G^{(n)}(p)$. For, let $d^{\prime}$ be the distance between $C f^{-1}\left\{G^{(n)}(p)\right\}$ and $f^{-1}(p)$, and let $m$ an integer such that $\frac{3}{m}<d^{\prime}$. If $G^{(m)}(p) G^{(m)}(q) \neq \phi$, then $O_{0}^{(m)}(p) O_{0}^{(m)}(q) \neq \phi$. For any point $z$ of $O_{0}^{(m)}(q)$, there exists $y \in f^{-1}(q)$ such that $z \in O^{(m)}(y)$. Since $O_{0}^{(m)}(p)$ and $O_{0}^{(m)}(q)$ are open inverse sets, there exists a point $r$ such that $f^{-1}(r) \subset O_{0}^{(m)}(p) O_{0}^{(m)}(q)$. Then there exists a point $w$ of $O^{(m)}(y) f^{-1}(r)$. Hence $w \in O^{(m)}(x)$ for some point $x$ of $f^{-1}(p)$. For this point $x$, we can easily see that $z \in O^{(n)}(x)$. As $O_{0}^{(m)}(q) \subset O_{0}^{(n)}(p)$ follows from that $O_{0}^{(m)}(q)$ is an inverse open set, we get $G^{(m)}(q)$ $\subset G^{(n)}(p)$.

By (1), (2) and A. H. Frink's theorem, ${ }^{4)} E$ is metrizable. This completes the proof of the theorem.

The following theorem due to A. V. Martin ${ }^{5)}$ is easily verified by use of Lemma 1 and Theorem 2 .

Theorem 3. If $S$ is a separable metric space and $f(S)=E$ is a closed continuous mapping such that $f^{-1}(p)$ is compact for each point $p$ of $E$, then $E$ is a separable metric space.

By Lemma 2 and Theorem 3, we have also the following:

4) A. H. Frink: Distance functions and the metrization problem, Bull. Amer. Math. Soc., 44, 133-142 (1937).

5) A. V. Martin: Loc. cit. 
Corollary. Let $S$ be a separable metric space and let $E$ a compact space. If $f(S)=E$ is a closed mapping such that $f^{-1}(p)$ is compact for each point $p$ of $E$, then $E$ is a separable metric space.

Theorem 4. If $S$ is a locally compact metric space and $f(S)=E$ is a closed continuous mapping such that $f^{-1}(p)$ is compact for each point $p$ of $E$, then $E$ is a locally compact metric space.

Proof. By Theorem 2, $E$ is metrizable. Accordingly we need only to prove that $E$ is locally compact. For this purpose, let $p$ be any point of $E$ and let $U$ any open neighborhood of $p$, then $f^{-1}(U)$ is open in $S$. For each point $x$ of $f^{-1}(p)$, there exists an open neighborhood $O(x)$ of $x$ such that $f^{-1}(U) \supset \overline{O(x)}$ and $\overline{O(x)}$ is compact because $S$ is a locally compact metric space. Since $f^{-1}(p)$ is compact, there exist open neighborhoods $O\left(x_{i}\right)$ of finite points $x_{i}(i=1,2, \ldots, n)$ of $f^{-1}(p)$ respectively such that $f^{-1}(U) \supset \sum_{i=1}^{n} O\left(x_{i}\right) \supset f^{-1}(p)$ where each $O\left(x_{i}\right)$ satisfies the same condition as the above $O(x)$. Then $f^{-1}(U) \supset$ $\sum_{i=1}^{n} \overline{O\left(x_{i}\right)} \supset \sum_{i=1}^{n} O\left(x_{i}\right) \supset f^{-1}(p)$.

Now let $O \equiv\left(\sum_{i=1}^{n} O\left(x_{i}\right)\right)_{0}$, then $U \supset f(\bar{O}) \supset f(O) \ni p$ and $f(O)$ is open because $f$ is closed and continuous. As $\sum_{i=1}^{n} \overline{O\left(x_{i}\right)} \supset \bar{O}$ and $\sum_{i=1}^{n} \overline{O\left(x_{i}\right)}$ is compact, $\bar{O}$ is compact. Hence $f(\bar{O})=\overline{f(O)}$ is compact. This completes the proof of the theorem. 